\title{
Measuring of the Perceptibility and Acceptability in Various Color Quality Measures
}

\author{
Ari Kim, Hong-suk Kim, and Seung-ok Park* \\ Department of Physics, Daejin University, Pocheon 487-711, Korea
}

(Received May 31, 2011 : revised July 25, 2011 : accepted August 1, 2011)

\begin{abstract}
Perceptibility and acceptability are the most often used threshold units in the field of color science. The former refers to a just perceptible difference and the latter evokes a tolerable color difference. Such thresholds can be very significant to color quality control processes in the printing industry and should be defined in quality measure units. Optical density (OD) and color difference models are usually utilized as color quality measures and have been provided in a considerable number of commercial measuring devices such as spectrophotometers. However, their merits and traits are far less understood in the literature. The present study intends to evaluate performance of those color quality measures including the OD and widely known color difference models, e.g. $\Delta \mathrm{E}^{*}{ }_{\mathrm{ab}}, \Delta \mathrm{E}_{\mathrm{CMC}(\mathrm{l}: \mathrm{c})}$ and $\Delta \mathrm{E}^{*}{ }_{00}$. A set of psychophysical assessments were carried out in order to accumulate the perceptibility and acceptability thresholds data, and their central trend was compared with the color quality measure estimates.
\end{abstract}

Keywords: Perceptibility, Acceptability, Color quality measure, Optical density, Color difference model OCIS codes : (330.1720) Color vision; (330.1730) Colorimetry; (330.5020) Perception psychology

\section{INTRODUCTION}

The minimum amount of a color variance perceived by the visual system is referred to as threshold. Perceptibility and acceptability are the most often used threshold units in the field of color science. However, they have been confused in many cases [1]. Perceptibility can correlate to a just perceptible difference, which is a psychophysical measurement of observers' judging whether a difference exists. Acceptability judgment is another visual judgment about a given physical stimulus. One thing that can be distinguished from perceptibility is that observers interpret and compare the magnitude of the stimulus with that of its tolerance. Such thresholds can be very significant to color quality control processes in the printing industry and should be defined in quality measure units. Optical density (OD) and color difference models are usually utilized as color quality measures and have been provided in a considerable number of commercial measuring devices such as spectrophotometers. However, their merits and traits for both perceptibility and acceptability thresholds are far less understood in the literature.

Kim et al. [2] measured the acceptability sensitivity of human observers to the reflection OD variation using a simpler method of limits and proposed a computational model for predicting the acceptability threshold and sensitivity as a function of OD of given color patches. Their findings show that the OD threshold function can be explained by a $\log$ function of OD. The current study intends to measure both acceptability and perceptibility psychophysically and to define the data in those various color quality measures including OD, CIELAB color difference $\left(\Delta \mathrm{E}^{*} \mathrm{ab}\right)$, $\mathrm{CMC}$ color difference $\left(\Delta \mathrm{E}_{\mathrm{CMC}(\mathrm{l:c})}\right)$ and $\mathrm{CIEDE} 2000\left(\Delta \mathrm{E}^{*}{ }_{00}\right)$; therefore performance of the quality measures can also be evaluated and compared with each other.

\section{BACKGROUNDS}

\subsection{Optical Density}

Densitometry has been widely used for color quality evaluations in the printing industry due to the strong linearity regarding colorant amounts and to simplicity. Based upon the Beer-Lambert law [3], optical density (OD) can be defined as

\footnotetext{
*Corresponding author: sopark@daejin.ac.kr

Color versions of one or more of the figures in this paper are available online.
} 

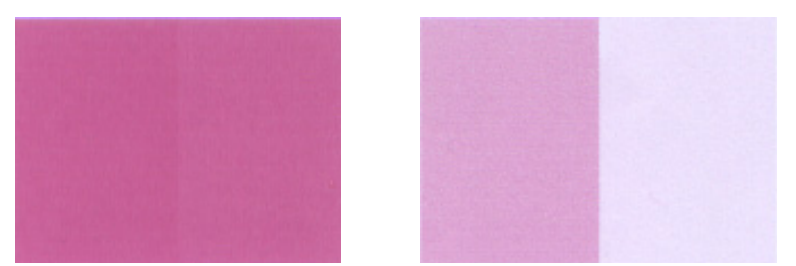

FIG. 1. Demonstration of perceptible color difference in (a) a high density color pair: 1.2 and 1.0 of OD and (b) a low density color pair: 0.25 and 0.05 of OD.

$$
\begin{aligned}
& O D=\log _{10}(1 / T) \\
& O D=\log _{10}(1 / R)
\end{aligned}
$$

where $\mathrm{T}$ and $\mathrm{R}$ denote transmittance and reflectance, respectively.

There are two types of density measurements: transmission and reflection. The former measures the density of transmissive or transparent samples and the latter measures that of reflected light through an optical filter that transmits a region of the light in one of the red, green or blue regions of the visible spectrum for separating color signals. The OD can also be defined in spectral and colorimetric domains. The spectral density function can be achieved by taking a logarithm for the inverse of reflectance function of a given color. The colorimetric density refers to the logarithm of normalized tri-stimulus values and correlates highly to tone reproduction curves.

Figure 1 demonstrates a smaller perceptible color difference at high density colors in the left pair (a) and a larger perceptible difference at low density colors in the right pair (b). However, difference in OD for those two pairs is identical (0.2). This sometimes causes misconceptions and miscommunications because the tolerance level of OD has to be adjusted for the tonal level of the reference color sample. Besides, the hue variation is not taken into account.

\subsection{Color Difference Models}

\subsubsection{CIELAB Color Difference}

The CIELAB color difference has been widely used because of its simplicity. It can be computed as the Euclidean distance between two points in CIELAB color space. [4] (See Equation (2))

$$
\Delta E_{a b}^{*}=\sqrt{\left(\Delta L^{*}\right)^{2}+\left(\Delta a^{*}\right)^{2}+\left(\Delta b^{*}\right)^{2}}
$$

where $\Delta L^{*}, \Delta a^{*}$, and $\Delta b^{*}$ are the Euclidean distance between the batch and its standard in CIELAB color space. The CIELAB color space (as well as CIELUV) was agreed upon by the Commission Internationale de l' Éclairage (CIE) in 1976.

The other way to calculate the CIELAB color difference is using perceptual attributes, lightness $\left(\Delta L^{*}\right)$, chroma ( $\Delta$
$\left.C^{*}{ }_{a b}\right)$ and hue $\left(\Delta H^{*}{ }_{a b}\right)$ as defined in following Equation (3).

$$
\Delta E_{a b}^{*}=\sqrt{\left(\Delta L^{*}\right)^{2}+\left(\Delta C^{*}\right)^{2}+\left(\Delta H^{*}{ }_{a b}\right)^{2}}
$$

where $\Delta H^{*}{ }_{a b}$ is a Euclidean distance while $\Delta h_{a b}$ is a polar metric angle.

\subsubsection{CMC}

The Color Measurement Committee (CMC) of the Society of Dyers and Colorists defined a color difference equation referred to as CMC (l:c) based on the CIELAB color difference components $\Delta L^{*}, \Delta C^{*}{ }_{a b}$, and $\Delta H^{*}{ }_{a b}$. [5] It includes two parameters: lightness weighting $(l)$ and chroma weighting (c), allowing the users to weight the difference based on the ratio of $l: c$.

$$
\Delta E_{C M C(l: c)}=\sqrt{\left(\frac{\Delta L^{*}}{l S_{L}}\right)^{2}+\left(\frac{\Delta C^{*}{ }_{a b}}{c S_{C}}\right)^{2}+\left(\frac{\Delta H^{*}{ }_{a b}}{S_{H}}\right)^{2}}
$$

where $S_{L}=\frac{0.040975 L_{S}^{*}}{\left(1+0.01765 L_{S}^{*}\right)}, \quad$ if $\quad L_{S}^{*} \geq 16$

$$
\begin{array}{ll}
\text { but } \quad S_{L}=0.511, & \text { if } \quad L_{S}^{*}<16 \\
\text { and } \quad S_{C}=\frac{0.638+0.0638 C_{a b, S}^{*},}{\left(1+0.01315 C_{a b, S}^{*}\right)}, & S_{H}=S_{C}(T F+1-F)
\end{array}
$$

The terms $T$ and $F$ are given by

$$
F=\sqrt{\frac{\left(C_{a b, S}\right)^{4}}{\left(\left(C_{a b, S}\right)^{4}+1900\right)}}
$$

and

$$
\begin{aligned}
& T=0.36+\left|0.4 \cos \left(h_{a b, S}+35\right)\right|, \quad \text { if } h_{a b, S} \leq 164 \text { or } h_{a b, S} \geq 345 \\
& T=0.56+\left|0.2 \cos \left(h_{a b, S}+168\right)\right|, \quad \text { if } 164<h_{a b, S}<345
\end{aligned}
$$

The subscript $S$, as in $C_{a b, S}$, denotes that the terms $S_{L}, S_{C}$, $S_{H}, F$ and $T$ are computed using the CIELAB lightness, chroma and hue angle (in degrees) of the standard. The terms $S_{L}, S_{C}$ and $S_{H}$ define the lengths of the semi-axes of the tolerance ellipsoid at the position of the standard in CIELAB space in each of the three directions $\left(S_{L}\right.$ for lightness, $S_{C}$ for chroma and $S_{H}$ for hue). The ellipsoids were fitted to visual tolerances determined from psychophysical experiments and the semi-axes in the CMC $(l: c)$ formula are used to effectively convert these ellipsoids into spheres at each point in CIELAB space. The parametric terms $l$ and $c$ constitute an important feature of the formula. These parameters allow the relative tolerances of the lightness 
and chroma components to be modified. For the textile industry it was recommended that $l=c=1$ for perceptibility decisions, whereas for acceptability decisions it was recommended that $l=2$ with $c=1$. The reason for this difference is that it is considered that, in terms of acceptability, differences in lightness should be weighted to be half as important as differences in either chroma or hue. The CMC (l:c) formula has been widely used in a number of industries and was adopted, for example, as a British Standard (BS 6923) and an AATCC test method (AATCC 173).

\subsubsection{CIEDE2000}

The CIE has recently agreed on the CIEDE2000 color difference formula for the evaluation of small color differences. [6] It includes not only lightness, chroma and hue weighting functions, but also an interactive term between the chroma and hue differences for improving the performance for blue colors and a scaling factor for the CIELAB $a^{*}$ scale for improving the performance for colors close to the achromatic axis.

$$
\Delta E_{00}^{*}=\sqrt{\left(\frac{\Delta L^{\prime}}{k_{L} S_{L}}\right)^{2}+\left(\frac{\Delta C^{\prime}}{k_{C} S_{C}}\right)^{2}+\left(\frac{\Delta H^{\prime}}{k_{H} S_{H}}\right)^{2}+R_{T}\left(\frac{\Delta C^{\prime}}{k_{C} S_{C}}\right)\left(\frac{\Delta H^{\prime}}{k_{H} S_{H}}\right)}
$$

where

$$
\begin{aligned}
& S_{L}=1+\left[0.015\left(L^{\prime}-50\right)^{2}\right] /\left[20+\left(L^{\prime}-50\right)^{2}\right]^{1 / 2} \\
& S_{C}=1+0.045 C^{\prime} \\
& S_{H}=1+0.015 C^{\prime} T
\end{aligned}
$$

The terms $\Delta L^{\prime}, \Delta C^{\prime}$ and $\Delta H^{\prime}$ aregiven by

$$
\begin{aligned}
& \Delta L^{\prime}=L_{T}^{\prime}-L_{S}^{\prime} \\
& \Delta C^{\prime}=C_{T}^{\prime}-C_{S}^{\prime} \\
& \Delta H^{\prime}=2 \sqrt{C_{T}^{\prime} C_{S}^{\prime}} \sin \left(\Delta h^{\prime} / 2\right)
\end{aligned}
$$

where the subscripts $S$ and $T$ refer to the standard and trial, respectively, and where

$$
\begin{aligned}
& \Delta h^{\prime}=h_{T}^{\prime}-h_{S}^{\prime} \\
& L^{\prime}=L^{*} \\
& a^{\prime}=(1+G) a^{*} \\
& b^{\prime}=b^{*} \\
& C^{\prime}=\sqrt{a^{\prime 2}+b^{\prime 2}} \\
& h^{\prime}=\tan \left(b^{\prime} / a^{\prime}\right)
\end{aligned}
$$

The $G$ and $T$ terms are computed using

$$
\begin{aligned}
G= & 0.5-0.5 \sqrt{C_{a b}^{* 7} /\left(C_{a b}^{* 7}+25^{7}\right)} \\
T= & 1-0.17 \cos \left(h^{\prime}-30\right)+0.24 \cos \left(2 h^{\prime}\right)+0.32 \cos \left(3 h^{\prime}+6\right) \\
& -0.20 \cos \left(4 h^{\prime}-63\right)
\end{aligned}
$$

Finally, the rotation term $R_{T}$ is given by

$$
R_{T}=-\sin (2 \Delta \theta) R_{C}
$$

where $R_{C}=2 \sqrt{C^{\prime 7} /\left(C^{\prime 7}+25^{7}\right)}$ and $\Delta \theta=30 e^{-\left(\left(h^{\prime}-275\right) / 25\right)^{2}}$

\subsection{Perceptibility and Acceptability}

Perceptibility and acceptability are often confused. Perceptibility can correlate to a just perceptible difference, [7] which is a psychophysical measurement of observers' judging whether a difference exists. In order to quantify a just-perceptible difference, a visual colorimeter can be used. In two halves of the bipartite field, a color-matched pair is seen. Observers are asked to change the wavelength of one of them until a difference is first noticed. This measure is obtained without any interpretation of human observers and treats the eye as a null detector. In other words, a visual judgment made without interpreting its importance is known as a perceptibility judgment.

Acceptability judgment is another visual judgment about color difference. One thing that distinguishes it from perceptibility is that observers interpret the color difference when they are seen. For instance, green foliage in summer gradually becomes yellow in early autumn. Its alterations are not easily recognized, because observers become more tolerant, but suddenly its change can be visible. These are judgments of acceptability. As soon as a batch of material is either "passed" or "failed," or "accepted" or "rejected," an acceptability decision has been made. Thus, even if two pairs of colors show the same perceptibility, acceptability judgments can be different to each other. Berns [1] illustrated examples of this judgment and explained in great detail.

\section{EXPERIMENTAL SETUP}

\subsection{Preparation of Samples}

Three color scales of cyan, magenta and yellow are selected as the test samples. Each of three color scales is equally spaced with digital input values; the interval of digital input values is one from 0 to 255 . In total, 768 color samples were printed by a selected color inkjet printer (Epson STYLUS PHOTO 2200) on a substrate of the A4 sized Epson Premium Glossy Photo Paper. They were engineered to print out solid colors only. Each color scale contains a hundred color segments of which colorant amount varies from 0 to $100 \%$ and the maximum and minimum OD are provided in Table 1. As can be seen, the maximum OD reaches up to 2.49 for cyan, 2.20 for magenta and 2.22 for yellow. 
TABLE 1. Maximum and minimum OD of three ramp color scales

\begin{tabular}{c|c|c|c}
\hline \hline & Cyan & Magenta & Yellow \\
\hline Max OD (100\%) & 2.49 & 2.20 & 2.22 \\
\hline Min OD $(0 \%)$ & 0 & 0 & 0 \\
\hline
\end{tabular}

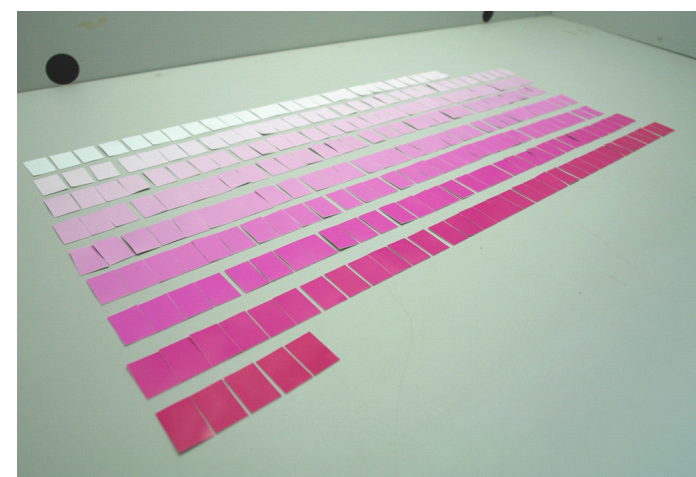

FIG. 2. Solid printed magenta scale samples.

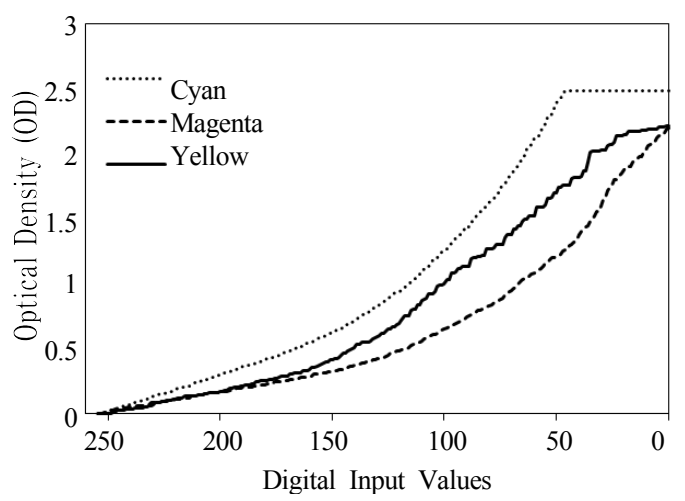

FIG. 3. Tone reproduction curves (TRCs) between digital input values and measured OD for three color scales (cyan, magenta and yellow).

Single color scale samples for magenta used in a current study is shown in Figure 2. Note that a step size of each color scale was manufactured to have a width of $20 \mathrm{~mm}$ and a height of $30 \mathrm{~mm}$.

Tone reproduction curves (TRCs) between the digital input values and measured OD are shown in Figure 3. The cyan scale was tone-clipped in digital input value of red from 0 to 50 and those colors were excluded in psychophysical assessments. In addition, OD of some colors were not different from their adjacent colors and so were also eliminated; therefore the number of test color samples used in psychophysical assessments can be 405 including 148 for cyan, 145 magenta and 112 for yellow.

\subsection{Instrumental Measurements}

A GretagMacbeth light booth was used to illuminate the test samples. $\mathrm{D}_{50}$ standard light source was chosen as a ref-

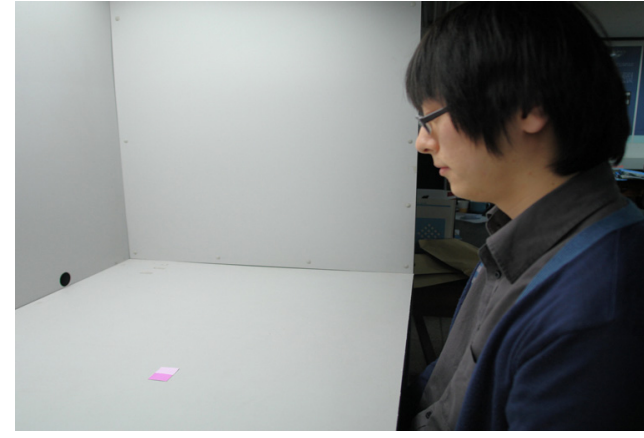

FIG. 4. Visual assessment with one of the observers.

erence white when the test samples were measured by spectrophotometer (GretagMacbeth SpectroEye) and tested by human observers. [8] For the accuracy, repeatability of the SpectroEye was tested. Ten successive measurements of all of the test colors used in this study were performed every 10 seconds for evaluating the short-term repeatability of the spectrophotometer. OD difference between the ten successive measurements was constant to 0 and mean $\Delta \mathrm{E}^{*}$ ab unit was approximately 0.2 .

\subsection{Visual Assessments}

In this psychophysical experiment, the perceptibility and acceptability thresholds were measured using method of limits [9] under 45/0 viewing geometry as illustrated in Figure 4. Five observers with normal color vision were required to assess each of solid cyan, magenta and yellow color scales covering a maximum tonal range of the color printer in both ascending and descending directions. According to Ref [11], for an ascending series, the experimenter presents a stimulus, beginning with one that is certain to be imperceptible, and asks the observers to respond 'yes' if they perceive it and 'no' if they do not. If they respond 'no', the experimenter increases the stimulus intensity and presents another trial. This continues until the observer responds 'yes'. A descending series begins with a stimulus intensity that is clearly perceptible and continues until the observers respond 'no' - that is, they cannot perceive the stimulus. Consequently, the thresholds are taken to be the mean values of both series in order to compensate for any adaptation effects. Similarly, the acceptability threshold can be measured by the accept/reject decisions [1] from the same observers. The average number of psychophysical assessments from a single observer was 2280 , and 6866 respectively for perceptibility and acceptability cases; thus 45730 pairs were evaluated by 5 observers in total.

\section{RESULTS AND DISCUSSION}

\subsection{Perceptibility Thresholds}

Figure 5 represents mean perceptibility thresholds in $\Delta$ OD unit for three color scales, cyan, magenta and yellow, 


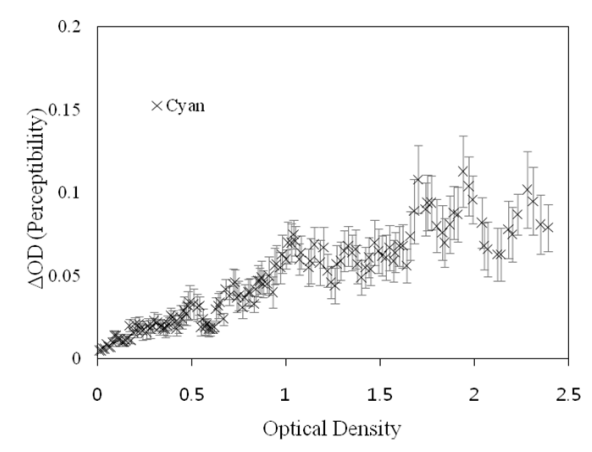

(a) Cyan

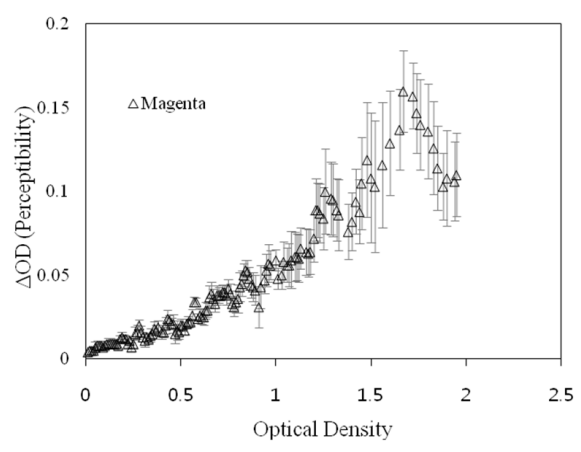

(b) Magenta

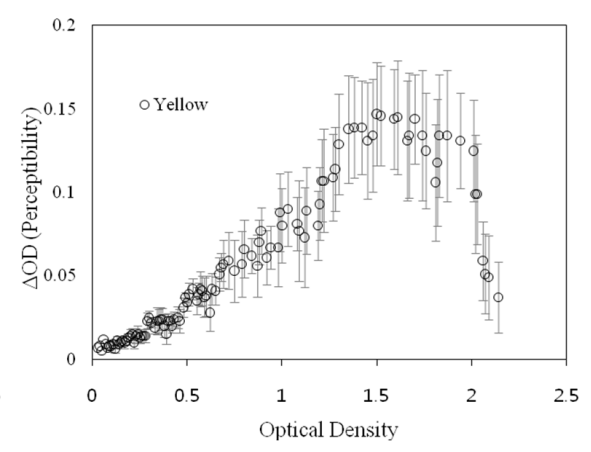

(c) Yellow

FIG. 5. Perceptibility: OD vs. mean perceptibility thresholds in OD unit for (a) cyan, (b) magenta and (c) yellow scales.
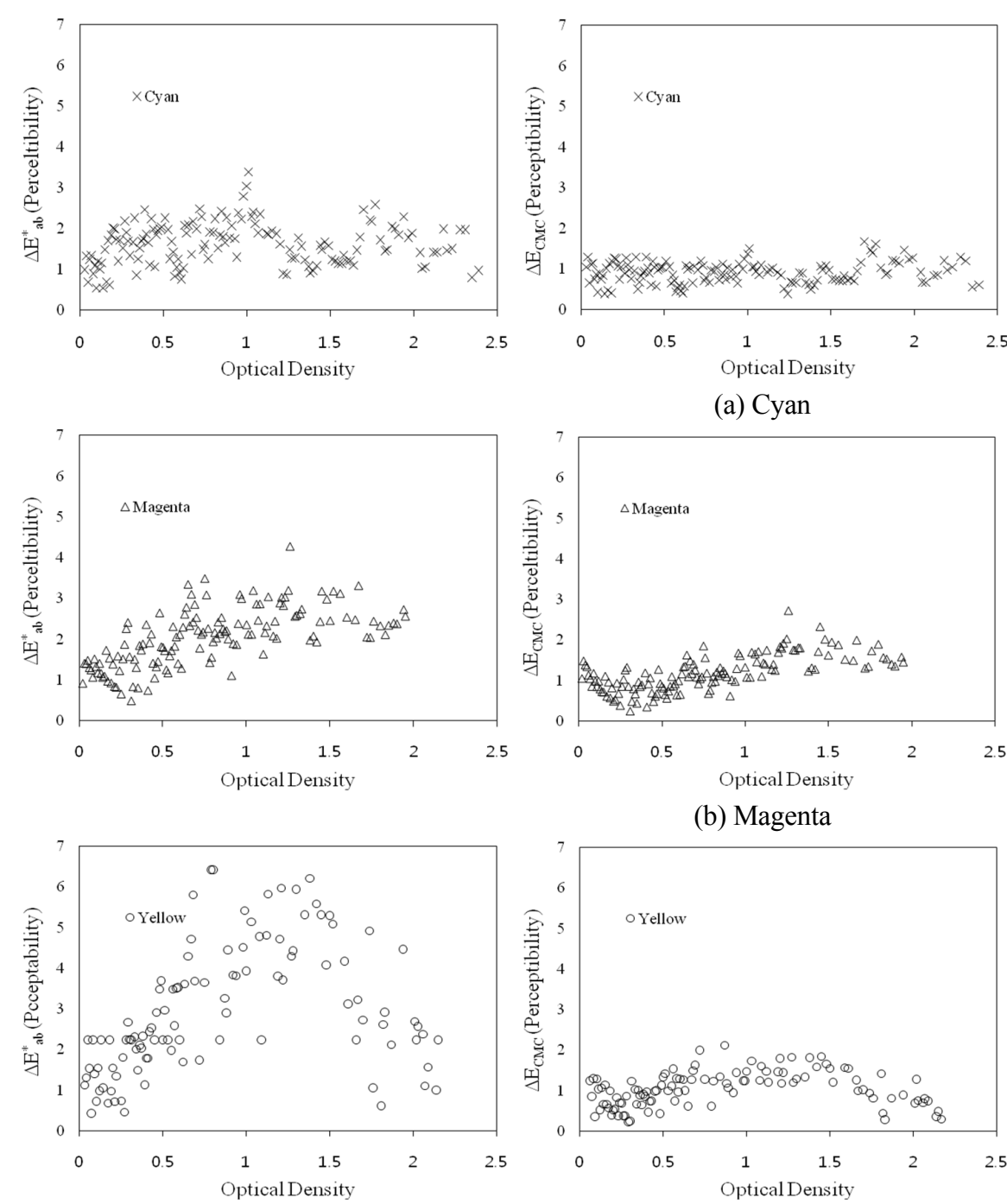

FIG. 6. Perceptibility: OD vs. perceptibility thresholds in $\Delta \mathrm{E}^{*}{ }_{\mathrm{ab}}$ (left), $\Delta \mathrm{E}_{\mathrm{CMC}(1: 1)}$ (center), and $\Delta \mathrm{E}^{*}{ }_{00}$ (right) units for (a) cyan, (b) magenta and (c) yellow scales.

across the varying OD from low to high. Reproducibility of psychophysical experiment can be estimated from standard error bars which express deviations between observers' (a) Cyan
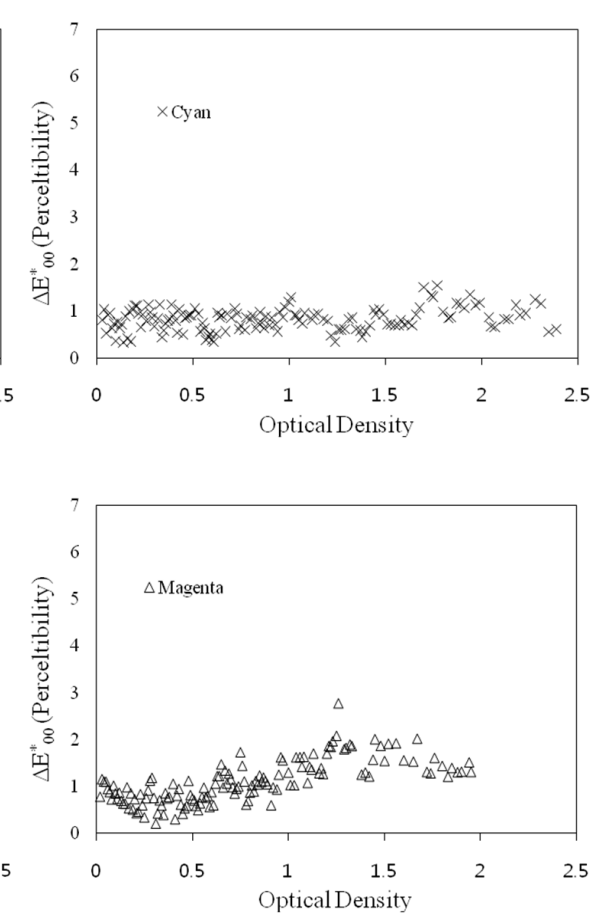

(b) Magenta
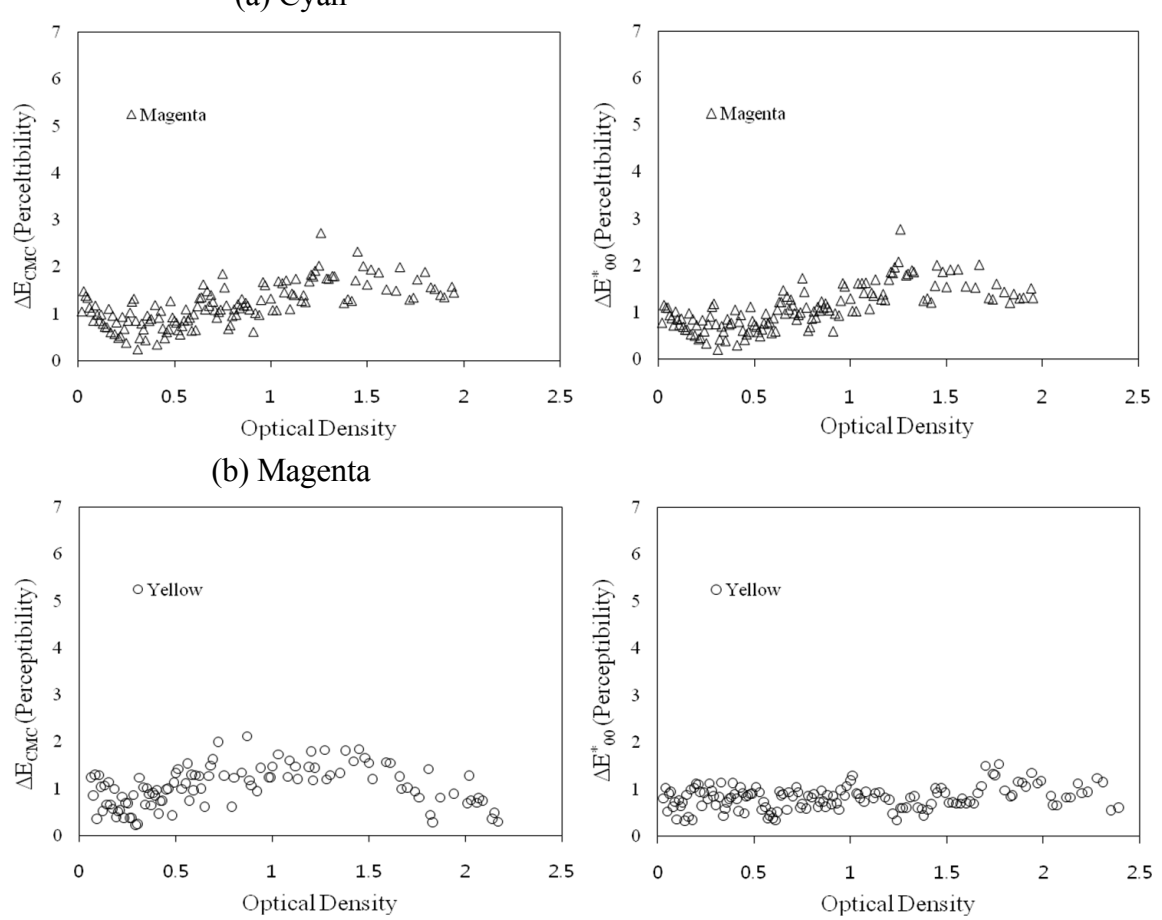

(c) Yellow 
responds more sensitively to the variation at the low-OD than high-OD fields. Moreover, it also confirmed that the $\triangle \mathrm{OD}$ does not correspond with human visual perception. Magenta and yellow scales show a similar basic trend. The threshold gradually increases as OD increases from 0 to 1.5 $\mathrm{OD}$, then it sharply decreases when OD increases to higher levels; therefore the curves appear to be bell shaped. However, for cyan, the bell shaped threshold function is not observed. The threshold fall-off was not measurable until the OD of 2.39. Density relies upon thickness of colorants used and on traversing the entire optical path so it does not account for any hue effects. However, color channel dependency was apparently observed for measuring $\triangle \mathrm{OD}$.

Figure 6 demonstrates the visually measured perceptibility thresholds in color difference models including $\Delta \mathrm{E}^{*}{ }_{\mathrm{ab}}, \Delta$

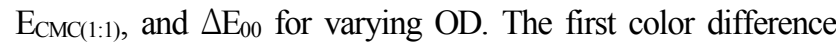
unit $\left(\Delta \mathrm{E}^{*}{ }_{\mathrm{ab}}\right)$ fluctuated for all colors, but became stable at 1 , when being defined in the rest $\left(\Delta \mathrm{E}_{\mathrm{CMC}(1: 1)}\right.$ and $\left.\Delta \mathrm{E}^{*}{ }_{00}\right)$. Note: the CMC model parameters of $1: 1$ were used for computing perceptibility thresholds as discussed in Section 2.2.2. Consequently, $\Delta \mathrm{E}^{*}{ }_{00}$ corresponds more to human visual perception compared to $\mathrm{OD}$ and $\Delta \mathrm{E}^{*}$ ab. In Table 2, minimum, maximum and average perceptibility thresholds defined in color difference model units along with their standard deviations are listed. Consequently, $\Delta \mathrm{E}_{\mathrm{CMC}(1: 1)}$ and $\Delta \mathrm{E}^{*}{ }_{00}$ correspond more to human visual perception compared to $\mathrm{OD}$ and $\Delta \mathrm{E}^{*}$ ab. The mathematical derivation of these color difference equations were previously given in Section 2.2.

\subsection{Acceptability Thresholds}

The acceptability threshold of OD which was visually measured for varying OD is shown in Figure 7. It provides the mean OD thresholds of both ascending and descending directions for cyan, magenta and yellow. The acceptability thresholds sharply increase as the OD increases in the low OD fields for the all colors. A slightly different trend was measured from the two different directions. Most threshold values for the ascending direction were higher than those of the descending direction. However, in both directions, the acceptability threshold increases as the OD increases in the low OD fields. Magenta and yellow scales show a similar basic trend. The threshold sharply increases as OD increases from 0 to $1.5 \mathrm{OD}$, then it was saturated when OD increased to higher levels. However, for cyan, it became constant at 0.3 from middle OD to high OD fields. Similar to the result for perceptibility threshold, channel dependency was also observed. Analogous to the previous

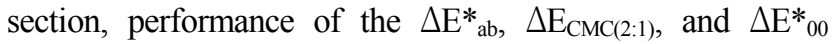
were evaluated with acceptability threshold data as illustrated in Figure 8. The first color difference unit $\left(\Delta \mathrm{E}^{*}{ }_{\mathrm{ab}}\right)$ fluctuated for all colors, but it became stable to a value around 3 , when being defined in the rest $-\Delta \mathrm{E}_{\mathrm{CMC}(2: 1)}$ and $\Delta \mathrm{E}^{*}{ }_{00}$. Note: the CMC model parameters of 2:1 were used for computing acceptability thresholds as discussed in Section 2.2.2. In Table 3, minimum, maximum and average perceptibility thresholds defined in color difference model units along with their standard deviations are listed.

\section{CONCLUSION}

In summary, the perceptibility and acceptability thresholds

TABLE 2. Minimum (Min), maximum (Max), average and standard deviation (STD) of perceptibility threshold in $\Delta \mathrm{E}^{*}{ }_{\mathrm{ab}}, \Delta \mathrm{E}_{\mathrm{CMC}(1: 1)}$ and $\Delta \mathrm{E}^{*}{ }_{00}$ units for cyan, magenta and yellow scales

\begin{tabular}{|c|c|c|c|c|c|c|c|c|c|c|c|c|}
\hline & \multicolumn{4}{|c|}{$\Delta \mathrm{E}_{\mathrm{ab}}^{*}$} & \multicolumn{4}{|c|}{$\Delta \mathrm{E}_{\mathrm{CMC}(1: 1)}$} & \multicolumn{4}{|c|}{$\Delta \mathrm{E}^{*}{ }_{00}$} \\
\hline & Min & Max & Average & STD & Min & Max & Average & STD & Min & Max & Average & STD \\
\hline Cyan & 0.55 & 3.39 & 1.64 & 0.52 & 0.38 & 1.67 & 0.91 & 0.26 & 0.33 & 1.54 & 0.82 & 0.24 \\
\hline Magenta & 0.48 & 4.28 & 2.04 & 0.71 & 0.24 & 2.72 & 1.19 & 0.44 & 0.20 & 2.76 & 1.10 & 0.45 \\
\hline Yellow & 0.43 & 6.42 & 2.94 & 1.55 & 0.22 & 2.11 & 1.04 & 0.43 & 0.19 & 1.54 & 0.79 & 0.29 \\
\hline
\end{tabular}

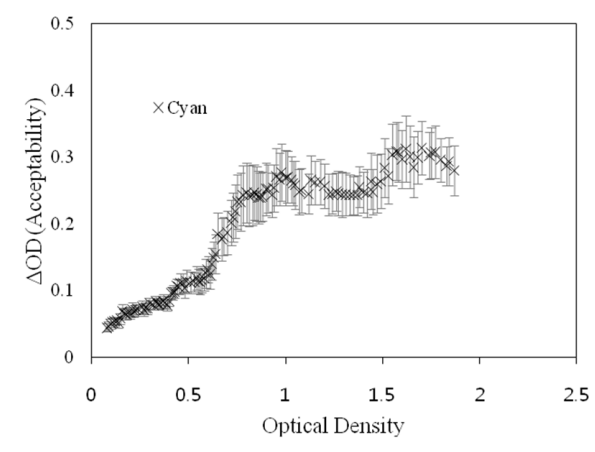

(a) Cyan

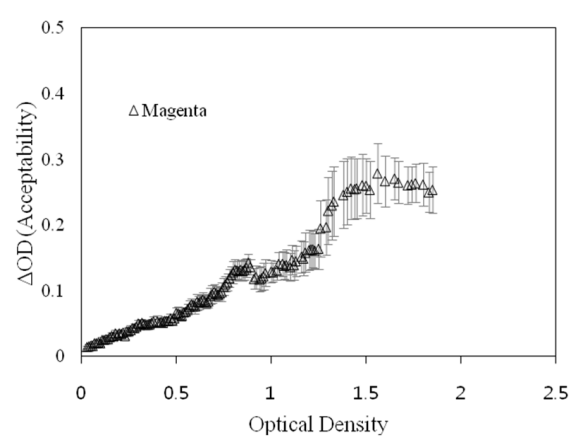

(b) Magenta

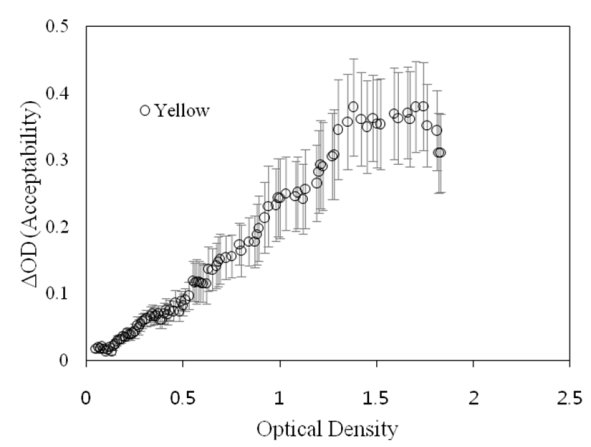

(c) Yellow

FIG. 7. Acceptability: OD vs. mean acceptability thresholds in OD unit for (a) cyan, (b) magenta and (c) yellow scales. 

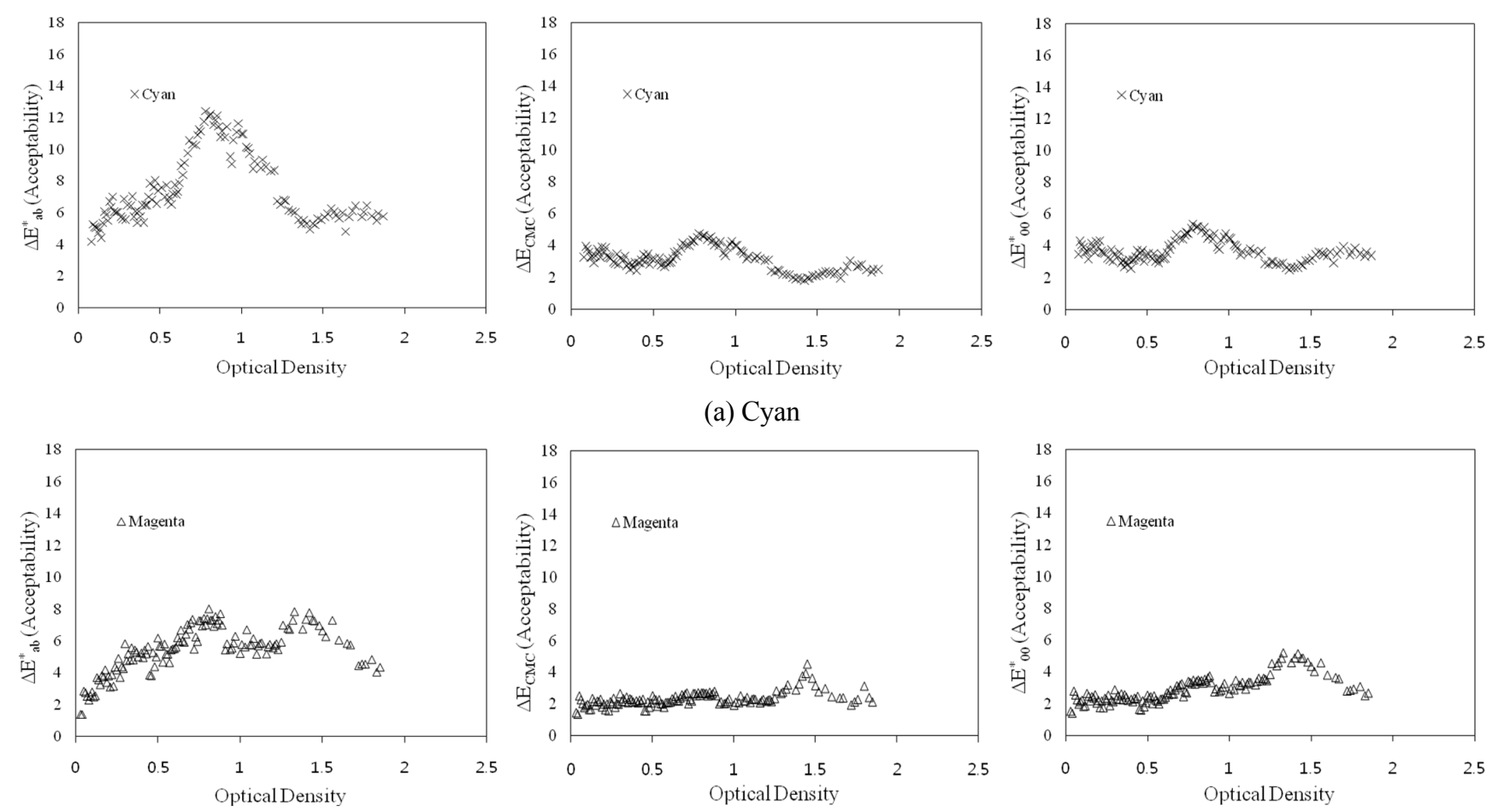

(b) Magenta
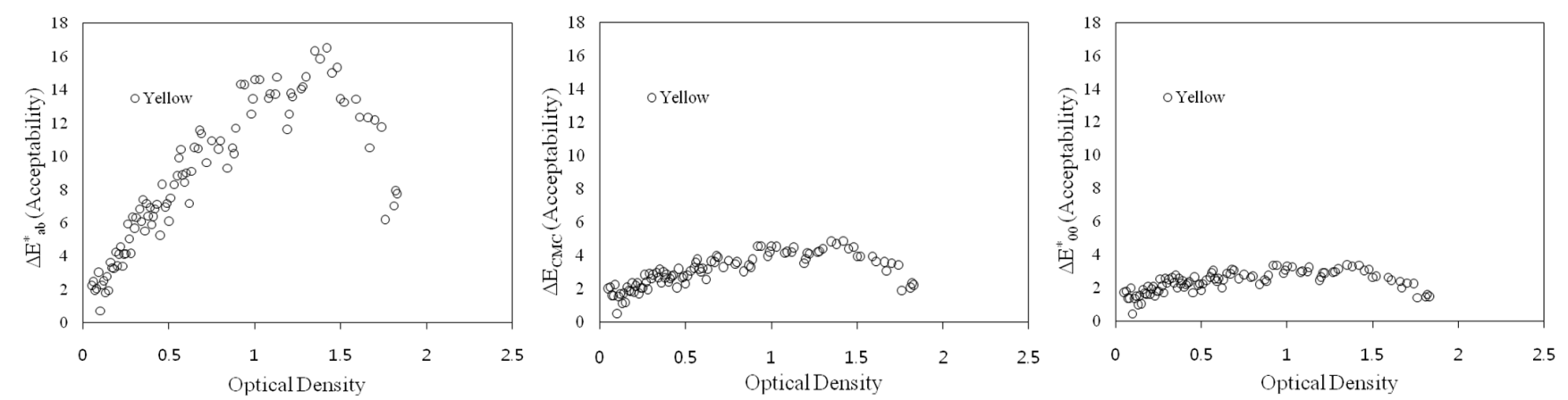

(c) Yellow

FIG. 8. Acceptability: OD vs. acceptability thresholds in $\Delta \mathrm{E}^{*}{ }_{\text {ab }}$ (left), $\Delta \mathrm{E}_{\mathrm{CMC}(2: 1)}$ (center), and $\Delta \mathrm{E}^{*}{ }_{00}$ (right) units for (a) cyan, (b) magenta and (c) yellow scales.

TABLE 3. Minimum (Min), maximum (Max), average and standard deviation (STD) of acceptability threshold in $\Delta \mathrm{E}^{*}$ ab, $\Delta \mathrm{E}_{\mathrm{CMC}(2: 1)}$ and $\Delta \mathrm{E}^{*}{ }_{00}$ units for cyan, magenta and yellow scales

\begin{tabular}{|c|c|c|c|c|c|c|c|c|c|c|c|c|}
\hline & \multicolumn{4}{|c|}{$\Delta \mathrm{E}_{\mathrm{ab}}^{*}$} & \multicolumn{4}{|c|}{$\Delta \mathrm{E}_{\mathrm{CMC}(2: 1)}$} & \multicolumn{4}{|c|}{$\Delta \mathrm{E}_{00}^{*}$} \\
\hline & Min & Max & Average & STD & Min & Max & Average & STD & Min & Max & Average & STD \\
\hline Cyan & 4.18 & 12.43 & 7.50 & 2.20 & 1.82 & 4.74 & 3.15 & 0.72 & 2.51 & 5.37 & 3.66 & 0.67 \\
\hline Magenta & 1.40 & 8.02 & 5.45 & 1.44 & 1.34 & 4.55 & 2.32 & 0.46 & 1.39 & 5.18 & 2.9 & 0.80 \\
\hline Yellow & 0.72 & 16.53 & 8.68 & 4.22 & 0.52 & 4.87 & 3.08 & 0.97 & 0.47 & 3.41 & 2.39 & 0.61 \\
\hline
\end{tabular}

across the reflection OD domain were psychophysically measured using the method of limits. The former steadily increases as the OD increases from the low to high density fields and it rapidly decreases at higher density fields. For the latter, the threshold sharply increases as OD increases from 0 to $1.5 \mathrm{OD}$ then it was saturated when OD increases to higher levels. Density relies upon the amount of ink used and traversing the entire optical path so it does not account for any hue effect. Furthermore, performance of three widely used color difference models, $\Delta \mathrm{E}^{*}{ }_{\mathrm{ab}}, \Delta \mathrm{E}_{\mathrm{CMC}(\mathrm{l}: \mathrm{c})}$ 
and $\Delta \mathrm{E}^{*}{ }_{00}$ were evaluated using our threshold results. The threshold in $\Delta \mathrm{E}^{*}$ ab unit fluctuated, but it become stable for the others $\left(\Delta \mathrm{E}_{\mathrm{CMCl} \text { (:c) }}\right.$ and $\left.\Delta \mathrm{E}^{*}{ }_{00}\right)$ for all colors. Consequently, the recent models such as $\Delta \mathrm{E}_{\mathrm{CMC}(\mathrm{l:c})}$ and $\Delta \mathrm{E}^{*}{ }_{00}$ appear more to correspond to human visual perception compared to OD and $\Delta \mathrm{E}^{*}{ }_{\mathrm{ab}}$. In future study, effects of viewing conditions such as viewing distance and surround luminance [10] on threshold perception of the visual system should be investigated in order to further extend the results.

\section{ACKNOWLEDGMENT}

This work was supported by the Daejin University Research Grants in 2011.

\section{REFERENCES}

1. R. S. Berns, "Measuring color quality: perceptibility and acceptability visual judgments," in Billmeyer and Saltzman's Principles of Color Technology, 3rd ed. (John Wiley \& Sons, NY, USA, 2000).

2. Y. J. Kim, S. K. Ha, H. C. Kim, Y. Bang, and H. Choh, "Measuring sensitivity of the visual system to reflection optical density variation," in Proc. 31th Conference of Korea Society for Imaging Science and Technology (Pu Kyong National Univ., Busan, Korea, Jun. 2009), pp. 90-91.

3. J. D. J. Ingle and S. R. Crouch, "Spectrochemical measurements," in Spectrochemical Analysis (Prentice Hall, New Jersey, USA, 1988).

4. CIE Publication 15.2, Colorimetry, 2nd ed. (Commission Internationale de l'Eclairage, Vienna, Austria, 1986).

5. F. J. J. Clarke, R. McDonald, and B. Rigg, "Modification to the JPC79 Colour-diffrence formula," J. Soc. Dyers \& Colourists 100, 117-148 (1984).

6. M. R. Luo, G. Cui, and B. Rigg, "The development of the CIE2000 color-difference formula: CIEDE2000," Col. Res. Appl. 26, 340-350 (1998).

7. P. Engeldrum, "Image quality modeling: where are we?," in Proc. IS\&T's PICS 1999 Conference (Springfield, Virginia, USA, 1999), pp. 251-255.

8. International Standards Organization, CIE Standard Illuminants for colorimetry, ISO 10526/CIE 5005-1999 (1999).

9. G. Gescheider, "The classical psychophysical methods," in Psychophysics: The Fundamentals, 3rd ed. (Lawrence Erlbaum Associates, NJ, USA, 1997).

10. Y. J. Kim and H. Kim, "Spatial luminance contrast sensitivity: effects of surround," J. Opt. Soc. Korea 14, 152-162 (2010).

11. M. D. Fairchild, Color Appearance Models, 2nd ed. (John Wiley \& Sons, West Sussex, England, 2005). 